\title{
CANAL SURFACES WITH GENERALIZED 1-TYPE GAUSS MAP
}

\author{
JINHUA QIAN, MENGFEI SU, AND YOUNG HO KIM
}

\begin{abstract}
This work considers a kind of classification of canal surfaces in terms of their Gauss map $\mathbb{G}$ in Euclidean 3-space. We introduce the notion of generalized 1-type Gauss map for a submanifold that satisfies $\Delta \mathbb{G}=f \mathbb{G}+g C$, where $\Delta$ is the Laplace operator, $C$ is a constant vector, and $(f, g)$ are non-zero smooth functions. First of all, we show that the Gauss map of any surface of revolution with unit speed profile curve in Euclidean 3-space is of generalized 1-type. At the same time, the canal surfaces with generalized 1-type Gauss map are discussed.
\end{abstract}

\section{INTRODUCTION}

In the 1970's, Chen introduced the notion of finite-type submanifolds in Euclidean space or pseudo-Euclidean space, which was extended to differential maps, in particular to Gauss maps of submanifolds. The notions of finite-type immersion and finite-type Gauss map are very useful tools in investigating and characterizing many important submanifolds [3, 1, 2. The simplest finite-type Gauss map is of 1-type, i.e., the Gauss map $\mathbb{G}$ of a submanifold $\mathbb{M}$ in Euclidean space or pseudoEuclidean space satisfies $\Delta \mathbb{G}=\lambda(\mathbb{G}+C)$ for some constant $\lambda \in \mathbb{R}(\lambda \neq 0)$ and a constant vector $C$, where $\Delta$ denotes the Laplace operator defined on $\mathbb{M}$, given by

$$
\Delta=-\frac{1}{\sqrt{\mathcal{G}}} \sum_{i, j} \frac{\partial}{\partial x^{i}}\left(\sqrt{\mathcal{G}} \tilde{g}^{i j} \frac{\partial}{\partial x^{j}}\right),
$$

where $\left(x_{1}, \ldots, x_{n}\right)$ is a local coordinate system of $\mathbb{M}, \tilde{g}^{i j}$ the components of the inverse matrix of the first fundamental form of $\mathbb{M}$, and $\mathcal{G}$ the determinant of the first fundamental form of $\mathbb{M}$. Planes, circular cylinders, and spheres in Euclidean 3-space are typical examples of surfaces with 1-type Gauss maps [12].

As a generalization of 1-type Gauss map, the definition of submanifold with pointwise 1-type Gauss map is proposed in $[9$, which takes the form $\Delta \mathbb{G}=f(\mathbb{G}+C)$

2020 Mathematics Subject Classification. 53A05, 53B25.

Key words and phrases. canal surface, Gauss map, generalized 1-type, Laplace operator.

The first author was supported by NSFC (no. 11801065) and the Fundamental Research Funds for the Central Universities (N2005012). The third author was supported by the Basic Science Research Program through the National Research Foundation of Korea (NRF) funded by the Ministry of Education (NRF-2020R1I1A3051852). 
for a non-zero smooth function $f$ and a constant vector $C$. For example, a helicoid, a catenoid, and a right cone in Euclidean 3-space are typical examples of surfaces with pointwise 1-type Gauss maps. Some related works have been done; for example, surfaces of revolution with pointwise 1-type Gauss map were studied in [5], and ruled submanifolds and hypersurfaces of Euclidean space with pointwise 1-type Gauss map were discussed in [6, 7, 9].

Very recently, the authors of 12 considered a cylindrical surface parameterized by

$$
x(s, t)=\left(\frac{s}{2} \cos (\ln s)+\frac{s}{2} \sin (\ln s),-\frac{s}{2} \cos (\ln s)+\frac{s}{2} \sin (\ln s), t\right),
$$

whose Gauss map satisfies

$$
\Delta \mathbb{G}=\frac{1}{s^{2}}(1+\cot (\ln s)) \mathbb{G}-\frac{1}{s^{2}} \csc (\ln s)(0,1,0) .
$$

This example yields the following definition.

Definition $1.1([12])$. The Gauss map $\mathbb{G}$ of a submanifold $\mathbb{M}$ in $\mathbb{E}^{m}$ is of generalized 1-type if the Gauss map $\mathbb{G}$ of $\mathbb{M}$ satisfies

$$
\Delta \mathbb{G}=f \mathbb{G}+g C
$$

for some non-zero functions $(f, g)$ on $\mathbb{M}$ and a constant vector $C$, where $\Delta$ denotes the Laplace operator defined on $\mathbb{M}$.

Indeed, the notion of generalized 1-type Gauss map can be regarded as a generalization of both 1-type Gauss map and pointwise 1-type Gauss map, since if both $f$ and $g$ are non-zero constants, then (1.1) can be written as $\Delta \mathbb{G}=\lambda(\mathbb{G}+C)$, $(0 \neq \lambda \in \mathbb{R})$. In this case, the Gauss map is just of 1 -type in the usual sense. If the function $f$ is equal to $g, 11.1$ can be expressed as $\Delta \mathbb{G}=f(\mathbb{G}+C)$, which is called the Gauss map of pointwise 1-type. More precisely, the pointwise 1-type Gauss map is said to be of the first kind when $C=0$, and of the second kind otherwise. If $f$ and $g$ vanish identically, then $\mathbb{G}$ is said to be harmonic.

Based on the definition of generalized 1-type Gauss map, the authors of $[12$ completely classified developable surfaces in Euclidean 3-space with a generalized 1-type Gauss map, i.e., cylindrical surfaces, conical surfaces, and tangent developable surfaces. Naturally, this idea of generalized 1-type Gauss map of submanifolds can be extended into many other submanifolds both in Euclidean space and in pseudo-Euclidean space.

The class of surfaces formed by sweeping a sphere was first investigated by Monge in 1850, who named them canal surfaces. Canal surfaces may be generated either by sweeping a sphere along a path, or by sweeping a particular circular cross-section of the sphere along the same path 8 . Canal surfaces are useful for representing long thin objects, e.g., pipes, poles, ropes, 3D fonts or intestines; they are also frequently used in solid and surface modelling for CAD/CAM. Representative examples are natural quadrics, tori, tubular surfaces, and Dupin cyclides. In 2016, the authors of [8] investigated the geometric properties of canal surfaces in Euclidean 3-space, and in 2019 they classified canal surfaces with pointwise 1-type Gauss map in [11. Based on the conclusions achieved in [11] and the definition of generalized 1-type 
Gauss map, we would like to discuss in this work the canal surfaces with generalized 1-type Gauss map.

This paper is organized as follows. In Section 2, some fundamental facts of canal surfaces are briefly recalled. In Section 3, surfaces of revolution and canal surfaces with generalized 1-type Gauss map are surveyed. Finally, some examples with generalized 1-type Gauss map are shown in Section 4.

Throughout this paper, we assume that all objects are smooth and all surfaces are connected, unless otherwise stated.

\section{Preliminaries}

Let $\mathbb{M}$ be a hypersurface in the Euclidean $(n+1)$-space $\mathbb{E}^{n+1}$. We denote the Levi-Civita connections of $\mathbb{E}^{n+1}$ and $\mathbb{M}$ by $\tilde{\nabla}$ and $\nabla$, respectively. Let $X, Y$ be vector fields tangent to $\mathbb{M}$ and let $\xi$ be a unit normal vector field of $\mathbb{M}$. Then the Gauss and Weingarten formulas are given respectively by

$$
\begin{aligned}
\tilde{\nabla}_{X} Y & =\nabla_{X} Y+h(X, Y), \\
\tilde{\nabla}_{X} \xi & =-A_{\xi} X .
\end{aligned}
$$

Here, $h$ is the second fundamental form and $A_{\xi}$ is the shape operator (or the Weingarten operator) in the direction of $\xi$ on $\mathbb{M}$. The eigenvalues and eigenvectors of $A_{\xi}$ are called the principal curvatures and principal directions of $\mathbb{M}$, respectively.

The mean curvature $H$ of $\mathbb{M}$ is defined by $H=\sqrt{\langle\vec{H}, \vec{H}\rangle}$, where $\vec{H}=\frac{1}{n} \operatorname{tr} h$ is the mean curvature vector field and $\operatorname{tr} h$ is the trace of $h$. The map $\mathbb{G}: \mathbb{M} \rightarrow \mathbb{S}^{n} \subset \mathbb{E}^{n+1}$ that maps each point of $\mathbb{M}$ to the unit normal vector to $\mathbb{M}$ at the point is called the Gauss map of $\mathbb{M}$, where $\mathbb{S}^{n}$ denotes the unit hypersphere centered at the origin of $\mathbb{E}^{n+1}$.

For a hypersurface in the Euclidean space $\mathbb{E}^{n+1}$, the following lemma plays an important role.

Lemma 2.1 (4]). Let $\mathbb{M}$ be an oriented hypersurface in the Euclidean space $\mathbb{E}^{n+1}$. Then the Laplacian of the Gauss map $\mathbb{G}$ of $\mathbb{M}$ satisfies

$$
\Delta \mathbb{G}=\left\|A_{\mathbb{G}}\right\|^{2} \mathbb{G}+n \nabla H,
$$

where $\left\|A_{\mathbb{G}}\right\|^{2}=\operatorname{tr}\left(A_{\mathbb{G}} A_{\mathbb{G}}\right)$ and $\nabla H$ is the gradient of the mean curvature $H$.

Note that, for a surface in $\mathbb{E}^{3}$, the gradient $\nabla H$ can be obtained by the following lemma.

Lemma 2.2 ([10]). Let $\mathbb{M}$ be an oriented surface in $\mathbb{E}^{3}$. Then the gradient $\nabla f$ of a smooth function $f$ defined on $\mathbb{M}$ can be formulated by

$$
\nabla f=\frac{1}{g_{11} g_{22}-\left(g_{12}\right)^{2}}\left\{\left(g_{22} f_{s}-g_{12} f_{t}\right) \partial_{s}+\left(-g_{12} f_{s}+g_{11} f_{t}\right) \partial_{t}\right\},
$$

where $\{s, t\}$ is a local coordinate system of $\mathbb{M}$ such that $\langle\partial s, \partial s\rangle=g_{11},\langle\partial s, \partial t\rangle=$ $g_{12}$, and $\langle\partial t, \partial t\rangle=g_{22}$, and $f_{s}, f_{t}$ are the partial derivatives of $f$ with respect to $s$ and $t$, respectively. 
In order to classify completely the canal surfaces with generalized 1-type Gauss map, we review some basic facts of canal surfaces in $\mathbb{E}^{3}$ according to [8].

A canal surface $\mathbb{M}$ in $\mathbb{E}^{3}$ is an immersed surface swept out by a sphere moving along an arbitrary curve $c=c(s)$ or by a particular circular cross-section of the sphere along the same path. Due to the generating process, the parametrization of $\mathbb{M}$ can be given as

$$
x(s, \theta)=c(s)+r(s)\{\cos \varphi(s) T(s)+\sin \varphi(s) \cos \theta N(s)+\sin \varphi(s) \sin \theta B(s)\},
$$

where $\theta \in[0,2 \pi), \varphi \in[0, \pi)$, and $-r^{\prime}(s)=\cos \varphi$ for some smooth function $\varphi(s)$. The curve $c(s)$ is called the spine curve (or center curve) and $r(s)$ is called the radial function of $\mathbb{M}$. In particular, if $c(s)$ is a straight line then $\mathbb{M}$ is a surface of revolution, and if $r(s)$ is a constant then $\mathbb{M}$ is a tube (or pipe surface). In what follows, $T, N, B$ are respectively the unit tangent, principal normal, and binormal vector fields defined along the spine curve $c$.

Initially, from (2.2) and the Frenet formula of a regular space curve $c$ in $\mathbb{E}^{3}$ with curvature $\kappa$ and torsion $\tau$, the quantities of the first fundamental form are given by

$$
\begin{aligned}
g_{11}= & \left\langle x_{s}, x_{s}\right\rangle \\
= & r^{2}\left(\kappa^{2} \sin ^{2} \varphi \cos ^{2} \theta+r^{\prime 2} \kappa^{2}+\tau^{2} \sin ^{2} \varphi+\varphi^{\prime 2}+2 \kappa \varphi^{\prime} \cos \theta+2 r^{\prime} \kappa \tau \sin \varphi \sin \theta\right) \\
& -\left(2 r r^{\prime \prime}+2 r \kappa \sin \varphi \cos \theta-\sin ^{2} \varphi\right), \\
g_{12}= & \left\langle x_{s}, x_{\theta}\right\rangle=r^{2} \tau \sin ^{2} \varphi+r^{2} r^{\prime} \kappa \sin \varphi \sin \theta, \\
g_{22}= & \left\langle x_{\theta}, x_{\theta}\right\rangle=r^{2} \sin ^{2} \varphi
\end{aligned}
$$

where

and

$$
x_{s}=\frac{\partial x}{\partial s}=x_{s}^{1} T+x_{s}^{2} N+x_{s}^{3} B, \quad x_{\theta}=\frac{\partial x}{\partial \theta}=x_{\theta}^{1} N+x_{\theta}^{2} B
$$

$$
\begin{aligned}
& x_{s}^{1}=\sin ^{2} \varphi-r r^{\prime \prime}-r \kappa \sin \varphi \cos \theta, \\
& x_{s}^{2}=r^{\prime} \sin \varphi \cos \theta-r r^{\prime} \kappa-r \tau \sin \varphi \sin \theta-r r^{\prime} \varphi^{\prime} \cos \theta, \\
& x_{s}^{3}=r^{\prime} \sin \varphi \sin \theta+r \tau \sin \varphi \cos \theta-r r^{\prime} \varphi^{\prime} \sin \theta, \\
& x_{\theta}^{1}=-r \sin \varphi \sin \theta, \quad x_{\theta}^{2}=r \sin \varphi \cos \theta .
\end{aligned}
$$

Then, we have

$$
g_{11} g_{22}-\left(g_{12}\right)^{2}=r^{2}\left(r r^{\prime \prime}+r \kappa \sin \varphi \cos \theta-\sin ^{2} \varphi\right)^{2} .
$$

Meanwhile, the Gauss map $\mathbb{G}$ of $\mathbb{M}$ is given by

$$
\mathbb{G}=\frac{x_{s} \times x_{\theta}}{\left\|x_{s} \times x_{\theta}\right\|}=\cos \varphi T+\sin \varphi \cos \theta N+\sin \varphi \sin \theta B,
$$

from which we have

$$
\begin{aligned}
\mathbb{G}_{s}= & -\left(r^{\prime \prime}+\kappa \sin \varphi \cos \theta\right) T-\left(r^{\prime} \kappa+r^{\prime} \varphi^{\prime} \cos \theta+\tau \sin \varphi \sin \theta\right) N \\
& +\left(\tau \sin \varphi \cos \theta-r^{\prime} \varphi^{\prime} \sin \theta\right) B, \\
\mathbb{G}_{\theta}= & -\sin \varphi \sin \theta N+\sin \varphi \cos \theta B .
\end{aligned}
$$


Based on 2.4 and 2.7), the shape operator of $\mathbb{M}$ is obtained as

$$
A_{\mathbb{G}}=\left(\begin{array}{cc}
-\frac{Q}{P} & \frac{R}{r P} \\
0 & -\frac{1}{r}
\end{array}\right)
$$

where

$$
\begin{aligned}
& P=r r^{\prime \prime}+r \kappa \sin \varphi \cos \theta-\sin ^{2} \varphi, \\
& Q=r^{\prime \prime}+\kappa \sin \varphi \cos \theta, \\
& R=\tau \sin ^{2} \varphi+r^{\prime} \kappa \sin \varphi \sin \theta .
\end{aligned}
$$

Note that, from 2.5$), P \neq 0$ everywhere due to the regularity of $\mathbb{M}$.

From (2.8), we see that the Gaussian curvature $K$ and the mean curvature $H$ are written, respectively, as

$$
K=\frac{Q}{r P}, \quad H=\frac{2 P+\sin ^{2} \varphi}{-2 r P} .
$$

At the same time, we have the following result.

Proposition 2.3 ([8]). The Gaussian curvature $K$ and the mean curvature $H$ of a canal surface $\mathbb{M}$ in $\mathbb{E}^{3}$ are related by

$$
H=-\frac{1}{2}\left(K r+\frac{1}{r}\right) .
$$

\section{MAin RESUlts}

In this section, we will focus on the surfaces of revolution and canal surfaces which have generalized 1-type Gauss maps.

3.1. Surfaces of revolution with generalized 1-type Gauss map. Let $\mathbb{M}$ be a surface of revolution in $\mathbb{E}^{3}$ parameterized by

$$
x(s, \theta)=(\psi(s), \phi(s) \cos \theta, \phi(s) \sin \theta)
$$

for some smooth functions $\psi$ and $\phi$. Without loss of generality, we assume that the profile curve is of unit speed, i.e., $\psi^{\prime 2}+\phi^{\prime 2}=1$. A direct computation shows that the Gauss map $\mathbb{G}$ of $\mathbb{M}$ is

$$
\mathbb{G}=\left(\phi^{\prime},-\psi^{\prime} \cos \theta,-\psi^{\prime} \sin \theta\right) .
$$

Then, the Laplacian $\Delta \mathbb{G}$ of the Gauss map $\mathbb{G}$ can be written as

$$
\Delta \mathbb{G}=-\frac{1}{\phi}\left(\phi \frac{\partial^{2} \mathbb{G}}{\partial s^{2}}+\phi^{\prime} \frac{\partial \mathbb{G}}{\partial s}+\frac{1}{\phi} \frac{\partial^{2} \mathbb{G}}{\partial \theta^{2}}\right) .
$$

Explicitly, it can be expressed as

$$
\Delta \mathbb{G}=\left(-\frac{\phi^{\prime} \phi^{\prime \prime}}{\phi}-\phi^{\prime \prime \prime},\left(\psi^{\prime \prime \prime}+\frac{\phi^{\prime} \psi^{\prime \prime}}{\phi}-\frac{\psi^{\prime}}{\phi^{2}}\right) \cos \theta,\left(\psi^{\prime \prime \prime}+\frac{\phi^{\prime} \psi^{\prime \prime}}{\phi}-\frac{\psi^{\prime}}{\phi^{2}}\right) \sin \theta\right) .
$$


Suppose that $\mathbb{M}$ has generalized 1-type Gauss map, i.e., $\Delta \mathbb{G}=f \mathbb{G}+g C$, where $C=\left(C_{1}, C_{2}, C_{3}\right)$ is a constant vector. Substituting $(3.2)$ and $(3.3)$ into (1.1), we obtain

$$
\left\{\begin{array}{l}
f \phi^{\prime}+g C_{1}=-\frac{\phi^{\prime} \phi^{\prime \prime}}{\phi}-\phi^{\prime \prime \prime} \\
f\left(-\psi^{\prime} \cos \theta\right)+g C_{2}=\left(\psi^{\prime \prime \prime}+\frac{\phi^{\prime} \psi^{\prime \prime}}{\phi}-\frac{\psi^{\prime}}{\phi^{2}}\right) \cos \theta \\
f\left(-\psi^{\prime} \sin \theta\right)+g C_{3}=\left(\psi^{\prime \prime \prime}+\frac{\phi^{\prime} \psi^{\prime \prime}}{\phi}-\frac{\psi^{\prime}}{\phi^{2}}\right) \sin \theta .
\end{array}\right.
$$

The second and third of these equations obviously imply that $C_{2}=C_{3}=0$. And

$$
\left\{\begin{aligned}
f(s) & =\frac{1}{\phi^{2}}-\frac{\phi^{\prime} \psi^{\prime \prime}}{\phi \psi^{\prime}}-\frac{\psi^{\prime \prime \prime}}{\psi^{\prime}} \\
g(s) & =\frac{1}{C_{1}}\left(-\phi^{\prime \prime \prime}-\frac{\phi^{\prime}}{\phi^{2}}-\frac{\phi^{\prime} \phi^{\prime \prime}}{\phi}+\frac{\psi^{\prime \prime} \phi^{2}}{\phi \psi^{\prime}}+\frac{\phi^{\prime} \psi^{\prime \prime \prime}}{\psi^{\prime}}\right) \\
& =\frac{1}{C_{1}}\left(-\phi^{\prime \prime \prime}-\frac{\phi^{\prime}}{\phi^{2}}+\frac{\psi^{\prime \prime}}{\phi \psi^{\prime}}+\frac{\phi^{\prime} \psi^{\prime \prime \prime}}{\psi^{\prime}}\right)
\end{aligned}\right.
$$

where $C_{1}$ is a non-zero constant.

Conversely, if we use the above information with the given functions $\psi$ and $\phi$, a surface of revolution with unit speed profile curve satisfies $\Delta \mathbb{G}=f \mathbb{G}+g C$ for such non-zero functions $(f, g)$ and a constant vector $C$. Thus, we have the following result.

Theorem 3.1. Any surface of revolution $\mathbb{M}$ with unit speed profile curve in $\mathbb{E}^{3}$ has generalized 1-type Gauss map. Explicitly, the Gauss map $\mathbb{G}$ of $\mathbb{M}$ satisfies

$$
\Delta \mathbb{G}=f \mathbb{G}+g C
$$

for some non-zero smooth functions $(f, g)$ given by (3.4) and a constant vector $C=\left(C_{1}, 0,0\right)$, where $C_{1}$ is a non-zero constant.

3.2. Canal surfaces with generalized 1-type Gauss map. In [11, the authors obtained the Laplacian of the Gauss map $\mathbb{G}$ for a canal surface $\mathbb{M}$. We will recall the relevant process as follows.

By (2.8) and (2.9), we have

$$
\left\|A_{\mathbb{G}}\right\|^{2}=\frac{r^{2} Q^{2}+P^{2}+R^{2}}{r^{2} P^{2}} \triangleq \frac{D}{r^{2} P^{2}} .
$$

From 2.10), the partial derivatives of mean curvature can be written as

$$
\begin{aligned}
H_{s}= & \frac{2 r^{2} r^{\prime} \kappa^{2} \sin ^{2} \varphi \cos ^{2} \theta-\left(2 r r^{\prime} \kappa-r^{2} \kappa^{\prime}\right) \sin ^{3} \varphi \cos \theta+5 r^{2} r^{\prime} r^{\prime \prime} \kappa \sin \varphi \cos \theta}{2 r^{2} P^{2}} \\
& +\frac{-2 r r^{\prime} r^{\prime \prime} \sin ^{2} \varphi+r^{2} r^{\prime \prime \prime} \sin ^{2} \varphi+4 r^{2} r^{\prime} r^{\prime \prime 2}+r^{\prime} \sin ^{4} \varphi}{2 r^{2} P^{2}}, \\
H_{\theta}= & -\frac{\kappa \sin ^{3} \varphi \sin \theta}{2 P^{2}} .
\end{aligned}
$$


By Lemma 2.2, 2.5) and 3.6, we get

$$
\nabla H(x)=\frac{1}{r^{2} P^{2}}\left[\left(x_{s}^{1} U\right) T+\left(x_{s}^{2} U+x_{\theta}^{1} V\right) N+\left(x_{s}^{3} U+x_{\theta}^{2} V\right) B\right],
$$

where we have put

$$
U=g_{22} H_{s}-g_{12} H_{\theta}, \quad V=-g_{12} H_{s}+g_{11} H_{\theta} .
$$

Substituting (2.6), 3.5), and (3.7) into 2.1) with $n=2$ in Lemma 2.1, we get

$$
\begin{aligned}
\Delta \mathbb{G}= & \frac{\left(D \cos \varphi+2 x_{s}^{1} U\right) T+\left[D \sin \varphi \cos \theta+2\left(x_{s}^{2} U+x_{\theta}^{1} V\right)\right] N}{r^{2} P^{2}} \\
& +\frac{\left[D \sin \varphi \sin \theta+2\left(x_{s}^{3} U+x_{\theta}^{2} V\right)\right] B}{r^{2} P^{2}} .
\end{aligned}
$$

Based on the above information, we have the following conclusions.

Theorem 3.2. An oriented canal surface $\mathbb{M}$ in $\mathbb{E}^{3}$ has generalized 1-type Gauss map if and only if it is one of the following surfaces:

(1) a surface of revolution such as

$$
x(s, \theta)=(r(s) \cos \varphi(s)+s, r(s) \sin \varphi(s) \cos \theta, r(s) \sin \varphi(s) \sin \theta),
$$

(2) a torus.

Proof. Suppose that an oriented canal surface $\mathbb{M}$ satisfies $\Delta \mathbb{G}=f \mathbb{G}+g C$. Without loss of generality, we may decompose the constant vector $C$ as

$$
C=C_{1} T+C_{2} N+C_{3} B,
$$

where $C_{1}=\langle C, T\rangle, C_{2}=\langle C, N\rangle, C_{3}=\langle C, B\rangle$.

Substituting (2.6), 3.9), and 3.10 into $\Delta \mathbb{G}=f \mathbb{G}+g C$, we obtain

$$
\left\{\begin{array}{l}
D \cos \varphi+2 x_{s}^{1} U=r^{2} P^{2}\left(f \cos \varphi+g C_{1}\right), \\
D \sin \varphi \cos \theta+2\left(x_{s}^{2} U+x_{\theta}^{1} V\right)=r^{2} P^{2}\left(f \sin \varphi \cos \theta+g C_{2}\right), \\
D \sin \varphi \sin \theta+2\left(x_{s}^{3} U+x_{\theta}^{2} V\right)=r^{2} P^{2}\left(f \sin \varphi \sin \theta+g C_{3}\right) .
\end{array}\right.
$$

From the first equation of 3.11 , we have the following two cases:

Case 1: $r^{\prime}=-\cos \varphi \neq 0$. In this case, we have

$$
f=\frac{D \cos \varphi+2 x_{s}^{1} U}{r^{2} P^{2} \cos \varphi}-\frac{g C_{1}}{\cos \varphi} .
$$

Taking (3.12) into the second and third equations of (3.11), we have

$$
\begin{aligned}
g & =\frac{2\left(x_{s}^{2} U+x_{\theta}^{1} V\right) \cos \varphi-2 x_{s}^{1} U \sin \varphi \cos \theta}{r^{2} P^{2}\left(C_{2} \cos \varphi-C_{1} \sin \varphi \cos \theta\right)} \\
& =\frac{2\left(x_{s}^{3} U+x_{\theta}^{2} V\right) \cos \varphi-2 x_{s}^{1} U \sin \varphi \sin \theta}{r^{2} P^{2}\left(C_{3} \cos \varphi-C_{1} \sin \varphi \sin \theta\right)} .
\end{aligned}
$$


According to 3.13 , we have

$$
\begin{aligned}
{\left[C_{3}\left(x_{s}^{2} U+x_{\theta}^{1} V\right)\right.} & \left.-C_{2}\left(x_{s}^{3} U+x_{\theta}^{2} V\right)\right] \cos ^{2} \varphi-x_{s}^{1} U \sin \varphi \cos \varphi\left(C_{3} \cos \theta-C_{2} \sin \theta\right) \\
& =C_{1} \sin \varphi \cos \varphi\left[\left(x_{s}^{2} U+x_{\theta}^{1} V\right) \sin \theta-\left(x_{s}^{3} U+x_{\theta}^{2} V\right) \cos \theta\right] .
\end{aligned}
$$

Rearranging (3.14) with the help of $(2.4)$ and $(3.8)$, we get

$$
\begin{aligned}
& \left(g_{22} H_{s}-g_{12} H_{\theta}\right) \\
& \quad \times\left[\left(\sin \varphi-r r^{\prime} \varphi^{\prime}-r \kappa \sin ^{2} \varphi \cos \theta\right)\left(C_{3} \cos \theta-C_{2} \sin \theta\right)-C_{3} r r^{\prime 2} \kappa-C_{1} r \tau \sin ^{2} \varphi\right. \\
& \left.\quad-r r^{\prime} \tau \sin \varphi\left(C_{3} \sin \theta+C_{2} \cos \theta\right)-C_{1} r r^{\prime} \kappa \sin \varphi \sin \theta\right] \\
& =\left(g_{11} H_{\theta}-g_{12} H_{s}\right)\left[r r^{\prime} \sin \varphi\left(C_{3} \sin \theta+C_{2} \cos \theta\right)+C_{1} r \sin ^{2} \varphi\right] .
\end{aligned}
$$

Because $\{\cos (m \theta), \sin (m \theta) \mid m \in \mathbb{N}\}$ forms a set of linearly independent functions, considering the coefficients of $\cos 4 \theta$ and $\sin 4 \theta$ in 3.15 with the help of (2.3) and (3.6), we have

$$
\left\{\begin{array}{l}
C_{2} r^{3} \kappa^{3} \sin ^{4} \varphi=0, \\
C_{3} r^{3} \kappa^{3} \sin ^{4} \varphi=0 .
\end{array}\right.
$$

From 3.16 , we consider the open subset $\mathcal{O}=\{p \in \mathbb{M} \mid \kappa(p) \neq 0\}$ of $\mathbb{M}$. Suppose that $\mathcal{O}$ is not empty. On $\mathcal{O}$, since $r \neq 0$ and $\sin \varphi \neq 0$, we have $C_{2}=C_{3}=0$. By (3.15), we have

$$
C_{1}\left[\left(g_{22} H_{s}-g_{12} H_{\theta}\right)\left(r^{\prime} \kappa \sin \theta+\tau \sin \varphi\right)+\left(g_{11} H_{\theta}-g_{12} H_{s}\right) \sin \varphi\right]=0 .
$$

Furthermore, by comparing the coefficients of the highest degree of the power of $\sin 3 \theta$ in 3.17 , we obtain that $C_{1}=0$, then $C=(0,0,0)$. In this situation, $\mathbb{M}$ has pointwise 1-type Gauss map of the first kind, i.e., $\Delta \mathbb{G}=f \mathbb{G}$. In this case, $\mathcal{O}$ is part of a catenoid or a surface of revolution, i.e., $\kappa=0$ (for the details, see [11]). Therefore, $\mathcal{O}$ is empty and $\kappa \equiv 0$, i.e., $\mathbb{M}$ is a surface of revolution. Simplifying 3.15 with the help of 2.3 and $(3.6)$, we have

$\left(C_{3} \cos \theta-C_{2} \sin \theta\right)\left(\sin \varphi-r \varphi^{\prime}\right)\left(-2 r r^{\prime} r^{\prime \prime} \sin ^{2} \varphi+r^{2} r^{\prime \prime \prime} \sin ^{2} \varphi+4 r^{2} r^{\prime} r^{\prime \prime 2}+r^{\prime} \sin ^{4} \varphi\right)=0$

At the same time, when $\kappa=0$, by $(3.6)$

$$
H_{s}=\frac{-2 r r^{\prime} r^{\prime \prime} \sin ^{2} \varphi+r^{2} r^{\prime \prime \prime} \sin ^{2} \varphi+4 r^{2} r^{\prime} r^{\prime \prime 2}+r^{\prime} \sin ^{4} \varphi}{2 r^{2} P^{2}}, \quad H_{\theta}=0 .
$$

Then equation 3.18 can be written as

$$
\left(C_{3} \cos \theta-C_{2} \sin \theta\right)\left(\sin \varphi-r \varphi^{\prime}\right) H_{s}=0 .
$$

Obviously, from this it follows that $H_{s}=0$ or $C_{2}=C_{3}=0$. (Note that $\sin \varphi-r \varphi^{\prime} \neq$ 0 or else $P=0$ and $\mathbb{M}$ is degenerate.)

Case 1.1: $H_{s}=0$. Due to $H_{\theta}=0$ with $\kappa=0, \mathbb{M}$ has constant mean curvature. From the conclusion in [1], $\mathbb{M}$ has pointwise 1-type Gauss map of the first kind. 
Case 1.2: $C_{2}=C_{3}=0$. From 3.11, we have

$$
\left\{\begin{array}{l}
f=\frac{D}{r^{2} P^{2}}+\frac{2 r^{\prime} U\left(\sin \varphi-r \varphi^{\prime}\right)}{r^{2} P^{2} \sin \varphi}, \\
g=\frac{2 U\left(\sin \varphi-r \varphi^{\prime}\right)}{C_{1} r^{2} P^{2} \sin \varphi} .
\end{array}\right.
$$

Since $P, D, U$ are all functions of $s$ when $\kappa=0,(3.19$ yields that the functions $f, g$ depend on $s$ only, i.e., $f=f(s), g=g(s)$. Then, simplifying (3.19) with the help of 3.5 , 3.6), and 3.8 , we get

$$
\left\{\begin{array}{l}
f(s)=\frac{1}{r^{2}}+r^{2} K^{2}+\frac{\tau^{2}\left(1-r^{\prime 2}\right)^{2} K^{2}-2 r r^{\prime} r^{\prime \prime} H_{s} K}{r^{\prime \prime 2}}, \\
g(s)=-\frac{2 r H_{s} K}{C_{1} r^{\prime \prime}}
\end{array}\right.
$$

It is obvious that $\mathbb{M}$ is a surface of revolution with generalized 1-type Gauss map. Without loss of generality, we may assume the center curve $c(s)=(s, 0,0)$ and $T=(1,0,0), N=(0,1,0), B=(0,0,1)$. Then, $\mathbb{M}$ can be represented by

$$
x(s, \theta)=(r(s) \cos \varphi(s)+s, r(s) \sin \varphi(s) \cos \theta, r(s) \sin \varphi(s) \sin \theta) .
$$

Comparing the above parametrization of $\mathbb{M}$ with the general form of the surface of revolution as stated in 3.1, we let $\psi(s)=r(s) \cos \varphi(s)+s, \phi(s)=r(s) \sin \varphi(s)$, and $\psi^{\prime 2}(s)+\phi^{\prime 2}(s)=1$, i.e.,

$$
\left(1-r^{\prime 2}-r r^{\prime \prime}\right)^{2}=1-r^{\prime 2}
$$

Solving the above differential equation, we get

$$
(r+c)\left(2 c r-c^{2}\right)^{\frac{1}{2}}=3 c s+c_{0}, \quad\left(c, c_{0} \in \mathbb{R}\right) .
$$

Via Mathematica, we get a real solution of $r(s)$ as follows:

$$
r(s)=-\frac{3 c^{2}}{\sqrt[3]{4} B}-\frac{B}{6 c \sqrt[3]{2}}-\frac{c}{2}
$$

where $A=-972 c^{4} s^{2}-648 c^{3} c_{0} s-108 c^{2} c_{0}^{2}-54 c^{6}, B=\left(A+\sqrt{-2916 c^{16}+A^{2}}\right)^{\frac{1}{3}}$.

At the same time, if we combine the expression forms of $(f, g)$ in 3.20 and (3.4) we have

$$
\left\{\begin{array}{l}
f(s)=\frac{1}{r^{2} \sin ^{2} \varphi}+\frac{\varphi^{\prime} \cos ^{2} \varphi}{r \sin ^{2} \varphi}-\frac{\varphi^{\prime \prime} \cos \varphi}{\sin \varphi}+\varphi^{\prime 2}, \\
g(s)=\frac{1}{C_{1}}\left(\frac{\varphi^{\prime \prime}}{\sin \varphi}-\frac{\cos \varphi}{r^{2} \sin ^{2} \varphi}-\frac{\varphi^{\prime} \cos \varphi}{r \sin ^{2} \varphi}\right) .
\end{array}\right.
$$

Thus, the Gauss map $\mathbb{G}$ of $\mathbb{M}$ is of generalized 1-type for some non-zero smooth functions $(f, g)$ given by $(3.23)$ and a constant vector $C=\left(C_{1}, 0,0\right)$, where $C_{1}$ is a non-zero constant.

Case 2: $r^{\prime}=-\cos \varphi=0$, i.e., $\mathbb{M}$ is a tube surface.

Suppose the interior $\mathcal{O}$ of the subset $\{p \in \mathbb{M} \mid \kappa(p)=0\}$ of $\mathbb{M}$ is non-empty, i.e., $r^{\prime}=0, \kappa=0$ on the open set $\mathcal{O}$. This means that $\mathcal{O}$ is part of a circular cylinder, and it has 1-type Gauss map of the first kind in the usual sense, i.e., the function $f$ 
is a non-zero constant 11. Therefore, $\mathcal{O}$ is empty. Thus, we may assume that $r^{\prime}=0, \kappa \neq 0$.

First of all, suppose that $C_{1} \neq 0$. Then, from the first equation of 3.11 we get

$$
g=\frac{2 x_{s}^{1} U}{C_{1} r^{2} P^{2}} .
$$

Taking (3.24) into the second and third equations of (3.11), we obtain

$$
\begin{aligned}
f & =\frac{D}{r^{2} P^{2}}+\frac{2\left(x_{s}^{2} U+x_{\theta}^{1} V\right)}{r^{2} P^{2} \sin \varphi \cos \theta}-\frac{2 C_{2} x_{s}^{1} U}{C_{1} r^{2} P^{2} \sin \varphi \cos \theta} \\
& =\frac{D}{r^{2} P^{2}}+\frac{2\left(x_{s}^{3} U+x_{\theta}^{2} V\right)}{r^{2} P^{2} \sin \varphi \sin \theta}-\frac{2 C_{3} x_{s}^{1} U}{C_{1} r^{2} P^{2} \sin \varphi \sin \theta} .
\end{aligned}
$$

According to this equation, we have

$$
C_{1}\left[\left(x_{s}^{2} U+x_{\theta}^{1} V\right) \sin \theta-\left(x_{s}^{3} U+x_{\theta}^{2} V\right) \cos \theta\right]=x_{s}^{1} U\left(C_{2} \sin \theta-C_{3} \cos \theta\right) .
$$

Simplifying (3.25) with the help of 2.3), 2.4 , 2.9), and (3.8), considering the coefficient of the power of $\sin \theta$ in 3.25$)$, we get $C_{1} r \kappa=0$, and hence $C_{1}=0$. This is a contradiction. Therefore, $C_{1}=0$. Together with the first equation of (3.11), we have $x_{s}^{1} U=0$, i.e.,

$$
\kappa^{\prime} \cos \theta+\kappa \tau \sin \theta=0
$$

Therefore, $\kappa=c(0 \neq c \in \mathbb{R})$ and $\tau=0$; then the center curve $c$ of $\mathbb{M}$ is a circle. This, together with the fact that $r$ is constant, implies that $\mathbb{M}$ is a torus.

Furthermore, from the second and third equations of 3.11 , we have

$$
\left\{\begin{aligned}
f & =\frac{D}{r^{2} P^{2}}+\frac{2 x_{\theta}^{1} V}{r^{2} P^{2} \sin \varphi \cos \theta}-\frac{g C_{2}}{\sin \varphi \cos \theta} \\
& =\frac{D}{r^{2} P^{2}}+\frac{2 x_{\theta}^{2} V}{r^{2} P^{2} \sin \varphi \sin \theta}-\frac{g C_{3}}{\sin \varphi \sin \theta}, \\
g & =\frac{2 V\left(x_{\theta}^{1} \sin \theta-x_{\theta}^{2} \cos \theta\right)}{r^{2} P^{2}\left(C_{2} \sin \theta-C_{3} \cos \theta\right)} .
\end{aligned}\right.
$$

Simplifying (3.26) with the help of $(2.3), 2.4),(2.9)$, and $(3.8)$, we have

$$
\left\{\begin{array}{l}
f=f(\theta)=4 H^{2}-2 K-\frac{4 r \kappa\left(H^{2}-K\right)\left(C_{2} \cos \theta+C_{3} \sin \theta\right) \sin \theta}{\left(C_{2} \sin \theta-C_{3} \cos \theta\right)}, \\
g=g(\theta)=\frac{4 r \kappa\left(H^{2}-K\right) \sin \theta}{C_{2} \sin \theta-C_{3} \cos \theta} .
\end{array}\right.
$$

Since $K, H$ are all functions of $\theta$ only when $r^{\prime}=0,(3.27)$ yields that the functions $(f, g)$ depend only on $\theta$. Therefore, the Gauss map $\mathbb{G}$ of $\mathbb{M}$ is of generalized 1-type for some non-zero smooth functions $(f, g)$ given by $(3.27)$ and a constant vector $C=\left(0, C_{2}, C_{3}\right)$, where $C_{2}^{2}+C_{3}^{2} \neq 0$. by

Conversely, suppose that $\mathbb{M}$ is a torus or a surface of revolution parameterized

$$
x(s, \theta)=(r(s) \cos \varphi(s)+s, r(s) \sin \varphi(s) \cos \theta, r(s) \sin \varphi(s) \sin \theta)
$$


satisfying 3.22 . One can easily check that $\Delta \mathbb{G}=f \mathbb{G}+g C$ is satisfied for some nonzero smooth functions $(f, g)$ given by $(3.23)$ and $(3.27)$ with the constant vectors $C=\left(0, C_{2}, C_{3}\right)$ and $C=\left(C_{1}, 0,0\right)$, respectively. This completes the proof.

As immediate consequences of the above theorem, we can easily get the following conclusions.

Corollary 3.3. Let an oriented canal surface $\mathbb{M}$ with generalized 1-type Gauss map be a surface of revolution. Then the Gauss map $\mathbb{G}$ of $\mathbb{M}$ satisfies

$$
\Delta \mathbb{G}=f \mathbb{G}+g C
$$

for some non-zero smooth functions $(f(s), g(s))$ given by 3.23 and a constant vector $C=\left(C_{1}, 0,0\right)$, where $C_{1}$ is a non-zero constant.

Corollary 3.4. Let an oriented canal surface $\mathbb{M}$ with generalized 1-type Gauss map be a torus. Then the Gauss map $\mathbb{G}$ of $\mathbb{M}$ satisfies

$$
\Delta \mathbb{G}=f \mathbb{G}+g C
$$

for some non-zero smooth functions $(f(\theta), g(\theta))$ given by (3.27) and a constant vector $C=\left(0, C_{2}, C_{3},\right)$, where $C_{2}, C_{3} \in \mathbb{R}$ and $C_{2}^{2}+C_{3}^{2} \neq 0$.

Remark 3.5. The canal surfaces with pointwise 1-type Gauss maps and the ones which have 1-type Gauss maps have been discussed in [11].

\section{EXAMPLES}

In this section, we present some examples with generalized 1-type Gauss map.

Example 4.1. Let $\mathbb{M}$ be a surface of revolution with unit speed profile curve, parameterized by

$$
x(s, \theta)=\left(\int \sin s^{2} d s, \int \cos s^{2} d s \cos \theta, \int \cos s^{2} d s \sin \theta\right) .
$$

Then, its Gauss map $\mathbb{G}$ is given by

$$
\mathbb{G}=\left(-\cos s^{2}, \sin s^{2} \cos \theta, \sin s^{2} \sin \theta\right) .
$$

The Laplacian $\Delta \mathbb{G}$ of the Gauss map $\mathbb{G}$ satisfies $\Delta \mathbb{G}=f \mathbb{G}+g C$ for smooth functions $f$ and $g$ as follows:

$$
\left\{\begin{array}{l}
f(s)=\frac{1}{\left(\int \cos s^{2} d s\right)^{2}}-\frac{2 s \cos ^{2} s^{2}}{\sin s^{2} \int \cos s^{2} d s}-\frac{2 \cos s^{2}}{\sin s^{2}}+4 s^{2}, \\
g(s)=\frac{\cos s^{2}}{C_{1}\left(\int \cos s^{2} d s\right)^{2}}-\frac{2 s \cos s^{2}}{C_{1} \sin s^{2} \int \cos s^{2} d s}-\frac{2}{C_{1} \sin s^{2}},
\end{array}\right.
$$

where $C=\left(C_{1}, 0,0\right), C_{1}$ a non-zero constant.

Example 4.2. Let $\mathbb{M}$ be a torus with $c(s)=(\sin s, 0, \cos s), r=\frac{1}{2}$, parameterized by

$$
x(s, \theta)=\left(\sin s-\frac{1}{2} \sin s \cos \theta, \frac{1}{2} \sin \theta, \cos s-\frac{1}{2} \cos s \cos \theta\right) .
$$

Then, its Gauss map $\mathbb{G}$ is given by

$$
\mathbb{G}=(\sin s \cos \theta,-\sin \theta, \cos s \cos \theta) .
$$


The Laplacian $\Delta \mathbb{G}$ of the Gauss map $\mathbb{G}$ satisfies $\Delta \mathbb{G}=f \mathbb{G}+g C$, where

$$
\left\{\begin{array}{l}
f(\theta)=\frac{8 \cos ^{2} \theta-16 \cos \theta+16}{(\cos \theta-2)^{2}}-\frac{8 \sin \theta\left(C_{2} \cos \theta+C_{3} \sin \theta\right)}{(\cos \theta-2)^{2}\left(C_{2} \sin \theta-C_{3} \cos \theta\right)}, \\
g(\theta)=\frac{8 \sin \theta}{(\cos \theta-2)^{2}\left(C_{2} \sin \theta-C_{3} \cos \theta\right)}
\end{array}\right.
$$

and $C=\left(0, C_{2}, C_{3}\right), C_{2}, C_{3} \in \mathbb{R}, C_{2}^{2}+C_{3}^{2} \neq 0$.

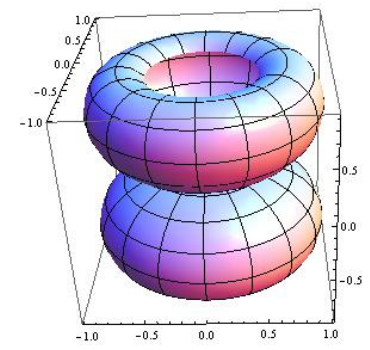

Figure 1. The surface of revolution in Example 4.1.

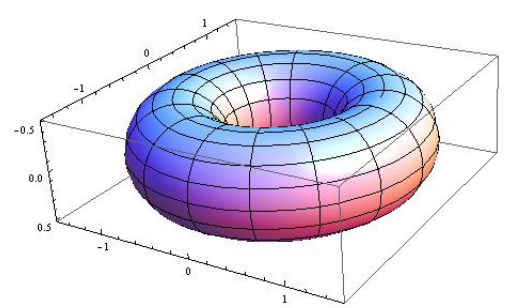

Figure 2. The torus in Example 4.2.

Example 4.3. Let $\mathbb{M}$ be a surface of revolution parameterized by

$$
x(s, \theta)=(r(s) \cos \varphi+s, r(s) \sin \varphi \cos \theta, r(s) \sin \varphi \sin \theta),
$$

where $r(s)$ satisfies (3.21), i.e., $(r+c)\left(2 c r-c^{2}\right)^{\frac{1}{2}}=3 c s+c_{0}\left(c, c_{0} \in \mathbb{R}\right)$. We put $c=1, c_{0}=0$; then $r(s)$ is given by

$$
r(s)=\frac{1}{2}\left(-1+\frac{1}{T}+T\right),
$$

where $T=\left(1+18 s^{\frac{1}{3}}+6 \sqrt{s^{2}+9 s^{4}}\right)^{\frac{1}{3}}$.

The surface $\mathbb{M}$ has generalized 1-type Gauss map as shown in Theorem 3.2 .

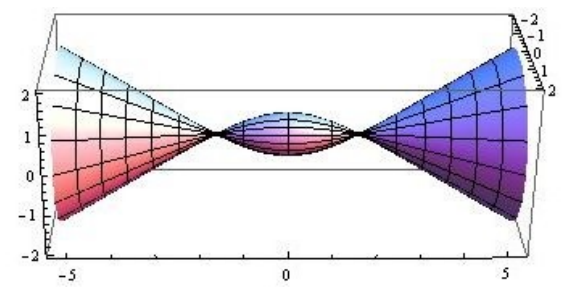

Figure 3. The surface of revolution in Example 4.3. 


\section{REFERENCES}

[1] C. Baikoussis, Ruled submanifolds with finite type Gauss map, J. Geom. 49 (1994), no. 1-2, 42-45. MR 1261104.

[2] C. Baikoussis, B.-Y. Chen and L. Verstraelen, Ruled surfaces and tubes with finite type Gauss map, Tokyo J. Math. 16 (1993), no. 2, 341-349. MR 1247657

[3] B.-Y. Chen, On submanifolds of finite type, Soochow J. Math. 9 (1983), 17-33. MR 0746741

[4] B.-Y. Chen and P. Piccinni, Submanifolds with finite type Gauss map, Bull. Austral. Math. Soc. 35 (1987), no. 2, 161-186. MR 0878431

[5] B.-Y. Chen, M. Choi and Y. H. Kim, Surfaces of revolution with pointwise 1-type Gauss map, J. Korean Math. Soc. 42 (2005), no. 3, 447-455. MR 2134709

[6] M. Choi and Y. H. Kim, Characterization of the helicoid as ruled surfaces with pointwise 1-type Gauss map, Bull. Korean Math. Soc. 38 (2001), no. 4, 753-761. MR 1865834

[7] U. Dursun, Hypersurfaces with pointwise 1-type Gauss map, Taiwanese J. Math. 11 (2007), no. 5, 1407-1416. MR 2368658

[8] Y. H. Kim, H. Liu and J. Qian, Some characterizations of canal surfaces, Bull. Korean Math. Soc. 53 (2016), no. 2, 461-477. MR 3483450

[9] Y. H. Kim and D. W. Yoon, Ruled surfaces with pointwise 1-type Gauss map, J. Geom. Phys. 34 (2000), no. 3-4, 191-205. MR 1762773

[10] J. Qian and Y. H. Kim, Classifications of canal surfaces with $L_{1}$-pointwise 1-type Gauss map, Milan J. Math. 83 (2015), no. 1, 145-155. MR 3347394.

[11] J. Qian and Y. H. Kim, Some classification of canal surfaces with the Gauss map, Bull. Malays. Math. Sci. Soc. 42 (2019), no. 6, 3261-3272. MR 3999089

[12] D. W. Yoon, D. S. Kim, Y. H. Kim and J. W. Lee, Hypersurfaces with generalized 1-type Gauss maps, Mathematics 6 (2018), no. 8, 130-144.https://doi.org/10.3390/math6080130

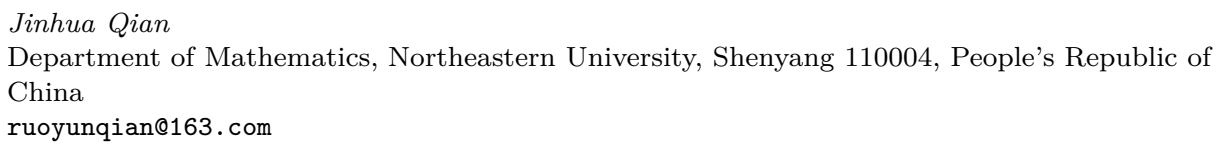

Mengfei Su

Department of Mathematics, Northeastern University, Shenyang 110004, People's Republic of China

mengfeisu@126.com

Young Ho Kim

Department of Mathematics, Kyungpook National University, Daegu 41566, Korea

yhkim@knu.ac.kr

Received: July 3, 2019

Accepted: April 21, 2020 